\title{
LE SEUIL DE RECONSTRUCTIBILITÉ PAR LE HAUT MODULO LA DUALITÉ DES RELATIONS BINAIRES FINIES
}

\author{
JAMEL DAMMAK \\ Université Claude Bernard Lyon 1, Tunisie
}

\begin{abstract}
Etant donnée une relation binaire $R$, de base $E$, on définit sa duale $R^{\star}$ par $R^{\star}(x, y)=R(y, x)$. La relation $R$ est dite auto-duale si elle est isomorphe $\grave{a} R^{\star}$. Une relation binaire $R^{\prime}$ est hémimorphe $\grave{a} R$, si elle est isomorphe $\grave{a} R$ ou $\grave{a} R^{\star}$. Une relation binaire $\grave{a}$ $n$ éléments est $(-k)$-demi-reconstructible, si elle est déterminée à l'hémimorphie près, par la donnée à l'hémimorphie près de ses restrictions de cardinal $(n-k)$. L'étude faite en [8] entraine la (-d)demi-reconstructibilité des relations binaires finies pour tout $d \geq 12$. Nous étabissons la $(-d)$-demi-reconstructibilité des relations binaires finies pour tout $d \in\{11,10,9,8,7,6\}$.

Given a binary relation $R$ of basis $E$, we define its dual $R^{\star}$ by $R^{\star}(x, y)=R(y, x)$. A relation $R$ is self-dual if it is isomorphic to $R^{\star}$. A binary relation $R^{\prime}$ is hemimorphic to $R$, if it is isomorphic to $R$ or to $R^{\star}$. A relation $R$ defined on $n$ elements is $(-k)$ half - reconstructible if it is determined, up to hemimorphism, by its restrictions of cardinality $(n-k)$. From [8] follows the $(-d)$-halfreconstructibility of finite binary relations, for all $d \geq 12$. We establish the $(-d)$-half-reconstructibility of finite binary relations, for all $d \in\{11,10,9,8,7,6\}$.
\end{abstract}

Mathematics Subject Classification : 03C60; 04A05; 05C20; 05C38; 05C40.

Key Words : Relation de différence, Relation binaire, Graphe, Hypomorphe, Hémimorphe, Reconstruction. 


\section{Introduction}

Soit $E$ un ensemble fini. Le cardinal de $E$ est noté $|E|$. Une relation binaire $R$ de base $E$ est une application du produit $E \times E$ dans $\{+,-\}$. Les éléments de $E$ sont appelés sommets de $R$. Les paires de sommets sont appelées arêtes de $R$. Une arête $\{a, b\}$ est dite neutre si $R(a, b)=R(b, a)$, elle est dite pleine (resp. vide) si $R(a, b)=R(b, a)=+(\operatorname{resp} . R(a, b)=R(b, a)=-)$. Une arête de $R$ est dite orientée, si elle n'est pas neutre, dans ce cas on dit que $a$ domine $b$ si $R(a, b)=+$. On appelle duale de $R$, la relation $R^{\star}$ définie sur $E$ par : pour tous éléments $x, y \in E, R^{\star}(x, y)=R(y, x)$. La relation $R$ est dite auto-duale si elle est isomorphe à $R^{\star}$. L'isomorphie entre deux relations $R$ et $R^{\prime}$ est notée $R \sim R^{\prime}$. Une relation binaire $T$ est un tournoi lorsque pour tout élément $x \in E, T(x, x)=-$ et pour tous éléments distincts $x, y \in E, T(x, y) \neq T(y, x)$. Un 3-cycle $(a, b, c)$ est un tournoi $T$ à 3 éléments défini par $T(a, b)=T(b, c)=T(c, a)=+$. Une $k$-chaîne est un ordre total irréflexif à $k$ éléments. La restriction de $R$ à une partie $X$ de $E$, notée $R / X$, est la relation de base $X$ définie par : pour tous éléments $x, y$ de $X, R / X(x, y)=R(x, y)$. Une relation $\mathrm{R}$ de base finie $E$ est dite connexe, si pour tous éléments distincts $x, y$ de $E$, il existe une suite $x_{0}=x, x_{1}, \ldots, x_{k}=y$, telle que pour tout $i=0,1, \ldots, k-1$, on a $R\left(x_{i}, x_{i+1}\right) \neq R\left(x_{i+1}, x_{i}\right)$.

Soient $R$ et $R^{\prime}$ deux relations binaires de bases respectives $E$ et $E^{\prime}$, et $f$ une bijection de $E$ sur $E^{\prime}$. L'application $f$ est dite un isomorphisme (resp. anti-isomorphisme) de $R$ sur $R^{\prime}$, si pour tous $x, y$ éléments de $E$, $R^{\prime}(f(x), f(y))=R(x, y)$ (resp. $\left.R^{\prime}(f(x), f(y))=R^{\star}(x, y)\right)$. Dans ce cas on dit que $R$ et $R^{\prime}$ sont isomorphes et on note $R^{\prime} \sim R$ (resp. $R$ et $R^{\prime}$ sont antiisomorphes et on note $\left.R^{\prime} \sim R^{\star}\right)$. Une relation binaire $R^{\prime}$ est hémimorphe à $R$, si elle est isomorphe à $R$ ou à $R^{\star}$. Une relation binaire $R$ est dite auto-duale si elle est isomorphe à $R^{\star}$. Une relation binaire est dite non auto-duale minimale, si elle est non auto-duale et si toutes ses restrictions strictes sont auto-duales. Une relation binaire $H$ s'abrite dans $R$ si $H$ est isomorphe à une restriction de $R$. Une restriction $R / A$ s'inverse dans $R^{\prime}$ si $R^{\prime} / A=R^{\star} / A$. Deux relations binaires $R$ et $R^{\prime}$ de base commune $E$ de cardinal $n$ sont dites $k$-hémimorphes (resp. $k$-hypomorphes) lorsque pour toute partie $X$ de $E$ de cardinal $k, R^{\prime} / X \sim R / X$ ou $R^{\prime} / X \sim R^{\star} / X$ (resp. $\left.R^{\prime} / X \sim R / X\right)$; définition analogue lorsque $\operatorname{card}(X) \leq k$, on remplace alors le préfixe $(k)$ par $(\leq k)$ dans les notations. La $(n-k)$-hémimorphie (resp. $(n-k)$-hypomorphie) est encore notée $(-k)$-hémimorphie (resp. $(-k)$-hypomorphie). Une relation $R$ est dite $k$-demi-reconstructible (resp. 
$k$-reconstructible) si toute relation $k$-hémimorphe (resp. $k$-hypomorphe) à $R$, lui est hémimorphe (resp. isomorphe); définition analogue pour la $(\leq k)$-demi-reconstructibilité (resp. ( $\leq k)$-reconstructibilité), et aussi de la $(-k)$-demi-reconstruction (resp. $(-k)$-reconstruction).

Dans ce papier nous montrons :

Théorème 3.1 Les relations binaires finies de cardinal $n \geq 23$ sont (n-11)-demi-reconstructibles.

Ensuite dans le cas général nous montrons : Pour tout entier $d \geq 6$, les relations binaires finies de cardinal $n \geq 16+d$ sont $(n-d)$-demireconstructibles.

\section{Définitions. Notations. Rappels.}

Somme lexicographique. Etant donnée une relation $S$ de base $I=$ $\{1, \ldots, k\}$, associons à chaque $i \in I$, une relation $R_{i}$ de base $I_{i}$ de telle sorte que les bases $I_{i}$ soient deux à deux disjointes. La $S$-somme des $R_{i}$, notée $S\left(R_{1}, \ldots, R_{k}\right)$, est la relation définie sur la réunion des $I_{i}$ de la façon suivante : $S\left(R_{1}, \ldots, R_{k}\right)(x, y)=R_{i}(x, y)$ si $x, y \in I_{i}$ et $S\left(R_{1}, \ldots, R_{k}\right)(x, y)=S(i, j)$ si $x \in I_{i}$ et $y \in I_{j}$ et $i \neq j$. Nous dirons aussi que la $S$-somme des $R_{i}$, est obtenue à partir de la relation $S$ en dilatant chaque $i \in I$ par la relation $R_{i}$.

Relations particulières. Citons les relations irréflexives particulières suivantes :

- Une presque-chaîne de longueur $k$ est obtenue à partir d'une $k$-chaîne en rendant neutre l'arête liant son premier et son dernier élément. Une presque-chaîne de longueur 3 est dite aussi une 3-consécutivité.

- Un pic est une relation à 3 éléments $a, b$ et $c$ telle que l'arête $\{a, b\}$ est neutre et les arêtes $\{a, c\},\{b, c\}$ sont orientées avec $R(a, c)=R(b, c)$.

- Un drapeau est une relation à 3 éléments $a, b$ et $c$ telle que l'arête $\{a, b\}$ est orientée, l'arête $\{b, c\}$ est pleine et l'arête $\{a, c\}$ est vide.

- Un diamant positif (resp. négatif) est un tournoi à 4 sommets constitué d'un 3-cycle $(a, b, c)$ et d'un point $d$ dominé par (resp. dominant) $(a, b, c)$. Le point $d$ est dit sommet du diamant.

- Etant donné un entier $h, T_{h}$ est le tournoi à $2 h+1$ sommets : $0,1, \ldots, 2 h$ tel que pour tout sommet $i, T_{h}(i, i+k)=+$ pour $k \in\{1, \ldots, h\}$, (l'entier $i+k$ étant considéré modulo $2 h+1)$. Une relation $R$ est un élément de 
$D\left(T_{h}\right)$, si $R$ est un tournoi obtenu en dilatant chaque sommet $k$ de $T_{h}$ par une chaîne $p_{k}$ de cardinal fini. Rappelons que $D\left(T_{h}\right)$ est la classe des tournois finis sans diamant ([7], [10]).

- Soient un entier naturel $h \geq 3$ et l'ensemble $F=\{1, \ldots, h\}$.

* On appelle consécutivité $1<2<\ldots<h$, l'une des quatre relations définies sur $F$ comme suit :

$\left[R_{1}(x, y)=+\operatorname{ssi}(y=x+1)\right],\left[R_{2}(x, y)=+\operatorname{ssi}(y=x+1\right.$ ou $\left.y=x)\right]$, $\left[R_{3}(x, y)=+\operatorname{ssi}(y=x+1\right.$ ou $\left.|y-x|>1)\right],\left[R_{4}(x, y)=+\operatorname{ssi}(y=\right.$ $x+1$ ou $|y-x| \neq 1)]$.

* On appelle cycle $1<2<\ldots<h<1$, l'une des quatre relations définies sur F comme suit : pour toute consécutivité $R_{i},(1 \leq i \leq 4)$, le cycle $R_{i}^{\prime}$ coïncide avec $R_{i}$, sauf peut être sur les couples $(1, h)$ et $(h, 1)$ où on a $R_{i}^{\prime}(h, 1)=+$ et $R_{i}^{\prime}(1, h)=-$.

- Etant donné un entier $n \geq 1$, on désigne par $S_{n}$, l'une des relations définies sur les $2 n$ éléments $t_{1}, \ldots, t_{2 n}$, par : $S_{n}\left(t_{i}, t_{i+n}\right)=S_{n}\left(t_{i+n}, t_{i}\right)$ pour $i=1, \ldots, n, S_{n}\left(t_{i}, t_{i+k}\right)=-S_{n}\left(t_{i+k}, t_{i}\right)=+$ pour $k=1, \ldots, n-1$ et pour $i=1, \ldots, 2 n$ (les entiers ici sont considérés modulo $2 n$ ). On notera $\delta_{n}$ un élément de la famille $E\left(S_{n}\right)$, des extensions de $S_{n}$ obtenues en augmentant la base de $S_{n}$ d'ensembles (éventuellement vides) deux à deux disjoints $s_{1}, \ldots, s_{2 n}$, appelés secteurs de la relation, tels que :

i) Pour tout $i, \delta_{n} / s_{i} \cup\left\{t_{i}, t_{i+1}\right\}$ est une chaîne de premier élément $t_{i}$ et de dernier élément $t_{i+1}$.

ii) Pour tout $i$, on a $\delta_{n} / s_{i} \cup s_{i+n}$ est un tournoi sans diamant.

iii) Pour tout $i$, pour tout $x$ de $s_{i}$, pour tout $y$ de $s_{i+j},(j=1, \ldots, n-1)$, on a $\delta_{n}(x, y)=-, \delta_{n}(y, x)=+, \delta_{n}\left(t_{i}, y\right)=-\delta_{n}\left(y, t_{i}\right)=+$, et pour tout $y$ de $s_{i+j},(j=n, \ldots, 2 n-1)$, on a $\delta_{n}\left(t_{i}, y\right)=-\delta_{n}\left(y, t_{i}\right)=-$.

Intervalle, Décomposabililité. La notion suivante d'intervalle d'une relation binaire a été introduite par R. Fraïssé en [6]. Etant donnée une relation binaire $R$ de base $E$, une partie $I$ de $E$ est un $R$-intervalle, lorsque pour tous éléments $a, b \in I$, tels que $R(a, a)=R(b, b)$, et pour tout élément $x \in E-I$, on a $R(a, x)=R(b, x)$ et $R(x, a)=R(x, b)$. Clairement, l'ensemble vide, les singletons et l'ensemble $E$ sont des intervalles de $R$, appelés intervalles triviaux. Une relation ayant au moins trois sommets sera dite indécomposable lorsque tous ses intervalles sont triviaux, elle est dit décomposable dans le cas contraire. Si $I$ et $J$ sont deux $R$-intervalles, la valeur $R(a, b)$ est une constante quand $a$ (resp. $b$ ) décrit l'ensemble $I$ (resp. $J$ ) et on note $R(I, J)$ cette constante.

Rappelons les résultats suivants : 
Lemme 2.1. [9] Les relations binaires finies sont $(\leq 6)$-reconstructibles.

Lemme 2.2. [5] Pour tout entier $d \geq 5$, les relations binaires connexes finies de cardinal $n \geq 7+d$ sont $(n-d)$-demi-reconstructibles.

Lemme 2.3. [4] Les relations binaires connexes finies sont $(\leq 7)$-demireconstructibles.

Lemme 2.4. [4] Pour tout entier $k \geq 2$, les relations binaires finies abritant un non auto-dual de cardinal $k$, sont $(\leq k+6)$-demi-reconstructibles.

Lemme 2.5. [12] Soient $R$ et $R^{\prime}$ deux relations binaires de même base $E$ de cardinal fini $n$. Si $R$ et $R^{\prime}$ sont q-hémimorphes (resp. q-hypomorphes) où $1 \leq q \leq n-1$, alors pour tout entier $p \leq \min (q, n-q)$, $R$ et $R^{\prime}$ sont p-hémimorphes (resp. p-hypomorphes).

Lemme 2.6. [2] Soit $B$ une relation binaire de base $\{1, \ldots, n\}$ (où $n \geq 2$ ), $i_{0} \in\{1, \ldots, n\}$, et $R$ (resp. $R^{\prime}$ ) la relation obtenu à partir du relation $B$, en dilatant le sommet $i_{0}$ par une relation $H$ (reps. $H^{\prime}$ ). Si les relations $R$ et $R^{\prime}$ sont isomorphes, alors les relations $H$ et $H^{\prime}$ sont aussi isomorphes.

Lemme 2.7. [1] Soient $n, d$ et $h$ des entiers naturels avec $1 \leq d \leq n-1$ et $1 \leq h \leq n-d, H$ une relation binaire à $h$ sommets, et $R$ et $R$ ' deux relations binaires $(n-d)$-hémimorphes et de base commune $E$ à $n$ éléments. Alors, pour toute partie $A$ de $E$ à au plus $d$ éléments, le nombre de parties $F$ de $E$ contenant $A$ telle que $R / F$ est hémimorphe à $H$, est égal au nombre de parties $F$ de $E$ contenant $A$ telle que $R^{\prime} / F$ est hémimorphe à $H$.

Notation. Dans la suite, on utilisera les notations suivantes (faites sous les hypothèses du lemme 2.7) $n(R, H, A)=\operatorname{card}\{F \subset E: A \subset F e t R / F \sim$ $\left.H o u R / F \sim H^{\star}\right\} n\left(R^{\prime}, H, A\right)=\operatorname{card}\left\{F \subset E: A \subset F e t R^{\prime} / F \sim H o u R^{\prime} / F \sim\right.$ $\left.H^{\star}\right\}$.

Sous ces notations, le lemme 2.7 dit que : $n(R, H, A)=n\left(R^{\prime}, H, A\right)$.

Lemme 2.8. ([5] et [11]). Soient $R$ et $R^{\prime}$ deux relations binaires de base commune $E$ de cardinal $n$.

i) Pour tout entier $h \geq 1$, si $R$ et $R^{\prime}$ sont $(4, n-h)$-hypomorphes et si $|E| \geq 6+h$, alors $R$ et $R^{\prime}$ sont isomorphes.

ii) Pour tout entier $h \geq 4$, si $R$ et $R^{\prime}$ sont $(n-h)$-hypomorphes et si $|E| \geq 6+h$, alors $R$ et $R^{\prime}$ sont isomorphes. 
Relation de différence. La notion de relation de différence a été introduite par G. Lopez dans [9]. Soient deux relations binaires $R$ et $R^{\prime}$ de même base $E$, qui sont $(\leq 2)$-hémimorphes. On définit la relation $D_{R, R^{\prime}}$ de base $E$ par : pour tout élément $x$ de $E, D_{R, R^{\prime}}(x, x)=+$ et pour tous éléments distincts $x, y$ de $E, D_{R, R^{\prime}}(x, y)=+$ lorsqu'il existe une suite $x=x_{0}, x_{1}, \ldots, x_{k}=y$ d'éléments de $E$ telle que $R\left(x_{i}, x_{i+1}\right) \neq R^{\prime}\left(x_{i}, x_{i+1}\right)$ pour tout $i$ élément de $\{0,1, \ldots, k-1\}$. La relation $D_{R, R^{\prime}}$ est une relation d'équivalence appelée relation de différence dont les classes sont appelées classes de différence.

Rappelons les résultats suivants :

Lemme 2.9. [10] Soient $R$ et $R^{\prime}$ deux relations binaires $(\leq 3)$-hypomorphes sur une même base finie $E$. Toute classe de l'équivalence $D_{R, R^{\prime}}$ est un intervalle commun à $R$ et $R^{\prime}$ sur lequel les restrictions de $R$ et $R$ ' sont réflexives ou irréflexives

Lemme 2.10. [10] Soient $R$ et $R^{\prime}$ deux relations binaires $(\leq 4)$-hypomorphes sur une base finie $E$, et $C$ une classe de l'équivalence $D_{R, R^{\prime}}$. Alors :

i) $\mathrm{Si} R / C$ est un tournoi, alors il existe un entier $h$ tel que $R / C$ est un $D\left(T_{h}\right)$.

ii) Si $R / C$ n'abrite pas de 3-cycle, alors $R / C$ est soit une chaîne, soit une presque-chaîne, soit une consécutivité, soit un cycle.

iii) Si $R / C$ abrite un 3-cycle, et si $R / C$ n'est pas un tournoi, alors il existe un entier $n$ tel que $R / C$ est un élément de $E\left(S_{n}\right)$.

Notons que comme conséquence du lemme 2.10, on a :

Corollaire 2.11. Soient $R$ et $R^{\prime}$ deux relations binaires $(\leq 4)$-hypomorphes sur une base finie $E$, et $C$ une classe de 1 'équivalence $D_{R, R^{\prime}}$. Alors :

i) S'il existe un entier $h$ tel que $R / C \in D\left(T_{h}\right)$, alors $R^{\prime} / C \in D\left(T^{\star}{ }_{h}\right)$ et $\left(R^{\prime} / C\right.$ et $\left.R^{\star} / C\right)$ sont $(\leq 6)$-hypomorphes.

ii) S'il existe un entier $n$ telle que $R / C$ est un élément de $E\left(S_{n}\right)$, alors $R^{\prime} / C \in E\left(S^{\star}{ }_{n}\right)$, et $\left(R^{\prime} / C\right.$ et $\left.R^{\star} / C\right)$ sont $(\leq 6)$-hypomorphes.

iii) $R / C$ et $R^{\prime} / C$ n'abritent pas de restrictions non auto-dual de cardinal $\leq 4$

Relation connexe et fortement connexe. Une relation $R$ de base finie $E$ est dite connexe (resp. fortement connexe), si pour tous éléments distincts $x, y$ de $E$, il existe une suite $x_{0}=x, x_{1}, \ldots, x_{k}=y$, telle que pour tout $i=0,1, \ldots, k-1$, on a $\left.R\left(x_{i}, x_{i+1}\right) \neq R\left(x_{i+1}, x_{i}\right)\right)\left(\operatorname{resp} . R\left(x_{i}, x_{i+1}\right)=\right.$ 
$-R\left(x_{i+1}, x_{i}\right)=+$ ). La composante connexe (rep. fortement connexe) d'une partie $A$ de $E$, telle que $R / A$ est connexe, est la plus grande partie $D$ de $E$ telle que $D$ contient $A$ et la restriction $R / D$ soit connexe (resp. $R / D$ soit fortement connexe).

Rappelons les résultats suivants :

Lemme 2.12. [5] Soient $d$ un entier $\geq 5, R$ et $R^{\prime}$ deux relations binaires $(-d)$-hémimorphes sur une base finie $E$ de cardinal $n$ au moins égal à $7+d$, et $C$ une classe de l'équivalence $D_{R, R^{\prime}}$. Si $C$ est différente de sa composante connexe, alors :

i) $C$ est un intervalle de $R$ et $R^{\prime}$ sur lequel les restrictions de $R$ et $R^{\prime}$ sont réflexives ou irréflexives.

ii) $R^{\prime} / C \sim R / C$.

Lemme 2.13. [5] Soient $R$ et $R^{\prime}$ deux relations binaires $(\leq 5)$-hémimorphes sur une base finie $E$. Si chacune des équivalences $D_{R, R^{\prime}}$ et $D_{R^{\star}, R^{\prime}}$ possède une seule classe, alors $R$ et $R^{\prime}$ sont des chaînes.

Lemme 2.14. [3] Soient $R$ et $R^{\prime}$ deux relations binaires $(\leq 5)$ - hémimorphes sur une base finie $E$ et $C$ une classe de l'équivalence $D_{R, R^{\prime}}$. Si $R / C$ et $R^{\prime} / C$ sont $(\leq 5)$-hypomorphes et si $R / C$ est un élément de $E\left(S_{n}\right)$, alors $R^{\prime} / C \sim R / C \sim R^{\star} / C$.

Notons que comme conséquence des lemme 2.10 et 2.14 on a :

Corollaire 2.15. Soient $R$ et $R^{\prime}$ deux relations binaires $(\leq 5)$ - hémimorphes sur une base finie $E, D$ une composante connexe de $R$ et $k$ le plus petit cardinal des restrictions non auto-duales de $R$. Si $k=6$ et si $R / D$ n'est pas un tournoi sans diamant, alors $R^{\prime} / D \sim R / D \sim R^{\star} / D$.

Corollaire 2.16. Soient $R$ et $R^{\prime}$ deux relations binaires $(\leq 5)$ - hémimorphes sur une base finie $E, D$ une composante connexe de $R$ et $k$ le plus petit cardinal des restrictions non auto-duales de $R$.

i) Si $k \in\{5,6\}$ et si $R / D$ n'est ni un tournoi sans diamant ni un élément de $E\left(S_{n}\right)$, alors $R^{\prime} / D \sim R / D \sim R^{\star} / D$.

ii) Si $k=6$ et si $R / D$ est un élément de $E\left(S_{n}\right)$, alors $R^{\prime} / D \sim R / D \sim$ $R^{\star} / D$.

Relations binaires non auto-duales minimales. Une relation binaire est dite non auto-duale minimale si elle est non auto-duale et toutes ses restrictions propres sont auto-duales.

Rappelons les résultats suivants : 
Lemme 2.17. [4] Soient $r \geq 2$ un entier, $R$ et $R^{\prime}$ deux relations binaires $(\leq 4)$-hémimorphes sur une base finie $E$. Si $R$ n'abrite aucune restriction non auto-duale à au plus $r$ sommets, alors les relations $R, R^{\star}$ et $R^{\prime}$ sont deux à deux $(\leq r)$-hypomorphes.

Lemme 2.18. [4] Soient $R$ et $R^{\prime}$ deux relations binaires $(\leq 5)$-hémimorphes sur une base finie $E$, et $C$ une classe de l'équivalence $D_{R, R^{\prime}}$, admettant une restriction non auto-duale, $k$ la plus petite cardinalité des restrictions non auto-duales de $R / C$ et $I_{0}$ la base d'une de ces restrictions. Alors la restriction $R^{\prime} / I_{0}$ est isomorphe à $R^{\star} / I_{0}$.

Notons que comme conséquence des lemmes 2.17 et 2.18 , on a :

Corollaire 2.19. Soit $r$ un entier, $2 \leq r \leq 6$. Soient $R$ et $R^{\prime}$ deux relations binaires $(\leq 5)$-hémimorphes sur une base finie $E$, et $C$ une classe de l'équivalence $D_{R, R^{\prime}}$. Alors $R / C$ et $R^{\prime} / C$ sont $(\leq r)$-hypomorphes ssi $R / C$ n'abrite aucune relation non auto-duale minimale à au plus $r$ sommets.

Lemme 2.20. [4] Soient $R$ et $R^{\prime}$ deux relations binaires $(\leq 6)$-hémimorphes sur une base finie $E$ admettant une restriction non auto-duale, $k$ la plus petite cardinalité des restrictions non auto-duales de $R, I_{0}$ la base d'une de ces restrictions et $C$ une classe de l'équivalence $D_{R, R^{\prime}}$. Si $R / I_{0} \sim R^{\prime} / I_{0}$, alors $C$ est un intervalle de $R$ et $R^{\prime}$, sur lequel les restrictions de $R$ et $R^{\prime}$ sont réflexives ou irréflexives.

Lemme 2.21. [4] Soient $R$ et $R^{\prime}$ deux relations binaires $(\leq 6)$-hémimorphes sur une base finie $E$, admettant une restriction non auto-duale, $C$ une classe de l'équivalence $D_{R, R^{\prime}}$ égale à sa composante connexe, $k$ la cardinalité minimale des restrictions non auto-duales qui s'abritent dans $R$, et $I_{0}$ la base d'une de ces restrictions. Si $R / I_{0} \sim R^{\prime} / I_{0}$, alors :

i) Si $R / I_{0}$ n'est pas un drapeau, alors $I_{0} \cap C=\phi$.

ii) Si $R / I_{0}$ est un drapeau, d'arête neutre $\{c, a\}$ et $\{c, b\}$, alors $I_{0} \cap C=\phi$ ou $I_{0} \cap C=\{c\}$.

Lemme 2.22. [4] Soient $d$ un entier $\geq 5, R$ et $R^{\prime}$ deux relations binaires $(\leq d)$-hémimorphes sur une base finie $E$ admettant une restriction non auto-duale, $k$ la cardinalité minimale des restrictions non auto-duales qui s'abritent dans $R, I_{0}$ la base d'une de ces restrictions et $C$ une classe de l'équivalence $D_{R, R^{\prime}}$.

i) $\mathrm{Si} C$ est différente de sa composante connexe, alors $R / C$ et $R^{\prime} / C$ sont $(\leq d-1)$-hypomorphes. 
ii) Si $d \geq 6$, si $R^{\prime} / I_{0} \sim R / I_{0}$, si $R / I_{0}$ un drapeau et si $C \cap I_{0} \neq \phi$, alors $R / C$ et $R^{\prime} / C$ sont $(\leq d-2)$-hypomorphes.

iii) Si $d \geq 6$ et si $R / I_{0} \sim R^{\prime} / I_{0}$, alors $R / C$ et $R^{\prime} / C$ sont $(\leq \max (k-$ $1, d-k))$-hypomorphes.

\section{La (-11)-demi-reconstructibilité des relations binaires finies.}

Théorème 3.1. Les relations binaires finies de cardinal $n \geq 23$ sont ( $n-$ 11)-demi-reconstructibles.

Soient $R$ et $R^{\prime}$ deux relations binaires $(n-11)$-hémimorphes sur une base finie $E$ de cardinal $n$ au moins égal à 23 et $k$ le plus petit cardinal des restrictions non auto-duales de $R$. Le lemme 2.5 prouve que les relations $R$ et $R^{\prime}$ sont $(\leq 11)$-hémimorphes. Suivant la valeur de $k$, on a:

-Si $R$ est connexe, alors le lemme 2.2 prouve que $R$ est $(n-11)$-demireconstructible.

- Si $2 \leq k \leq 5$, alors le lemme 2.4 prouve que $R$ est 11-demi-reconstructible et par suite $R$ est $(n-11)$-demi-reconstructible (lemme 2.5 ).

- Si $k \geq 7$ ou $k=0$. Dans ce cas $R$ n'admet aucune restriction non auto-duale de cardinal inférieur au égal à 6 , alors le lemme 2.1 prouve que $R$ est $(n-11)$-demi-reconstructible.

Dans la suite $k=6$, donc les relations $R, R^{\star}$ et $R^{\prime}$ sont deux à deux $(\leq 5)$-hypomorphes. Soit $D$ la plus grande composante connexe non autoduale de $R$. D'après le corollaire $2.15 R / D$ est un tournoi sans diamant, de même le lemme 2.13 prouve que l'une des équivalences $D_{R / D, R^{\prime} / D}$ ou $D_{R^{\star} / D, R^{\prime} / D}$ possède au moins deux classes.

La preuve du théorème 3.1 utilise les résultats suivants :

Proposition 3.2. Soient $D$ la plus grande composante connexe non autoduale de $R$. Si l'équivalence $D_{R / D, R^{\prime} / D}$ possède au moins deux classes, alors $R^{\prime} \sim R$.

Notons que comme conséquence de la proposition 3.2 on a :

Corollaire 3.3. Soient $D$ la plus grande composante connexe non autoduale de $R$. Si l'équivalence $D_{R^{\star} / D, R^{\prime} / D}$ possède au moins deux classes, alors $R^{\prime} \sim R^{\star}$. 
Dans la suite de la preuve de la proposition $3.2 C$ est une classe de l'équivalence $D_{R, R^{\prime}}$. Comme $k=6$, alors les relations $R$ et $R^{\prime}$ sont $(\leq 5)$ hypomorphes, et par suite d'après le lemme $2.9 C$ est un intervalle de $R$ et $R^{\prime}$, sur lequel les restrictions de $R$ et $R^{\prime}$ sont réflexives ou irréflexives, la preuve de la proposition 3.2 utilise les lemmes suivants :

Lemme 3.4. i) Si $C$ est différente de sa composante connexe, alors $R^{\prime} / C \sim$ $R / C$.

ii) Si $R / C$ n'est pas un tournoi sans diamant, alors $R^{\prime} / C \sim R / C$.

iii) Si $R / C$ est auto-dual, alors $R^{\prime} / C \sim R / C$.

Preuve. i) Si $C$ est différente de sa composante connexe, alors le lemme 2.12 prouve que $R^{\prime} / C \sim R / C$.

ii) On suppose que $C$ est une composante connexe de $R$. Comme $k=6$ et $R / C$ n'est pas un tournoi sans diamant, alors d'après le corollaire 2.2 $R^{\prime} / C \sim R / C$.

iii) Comme $R / C$ est connexe, alors le lemme 2.3 prouve que $R^{\prime} / C \sim$ $R / C$ ou $R^{\prime} / C \sim R^{\star} / C$. Si $R / C$ est auto-dual, alors $R^{\prime} / C \sim R / C \sim R^{\star} / C$.

Dans la suite on suppose que $C$ et $D$ sont deux composantes connexes non auto-duales de $R$ qui sont des tournois sans diamant.

Lemme 3.5. $R^{\prime} / D$ et $R / D$ sont $(\leq 6)$-hypomorphes.

Preuve. Puisque l'équivalence $D_{R / D, R^{\prime} / D}$ possède au moins deux classes et les relations $R$ et $R^{\prime}$ sont $(\leq 7)$-hémimorphes, alors le i) du lemme 2.22 prouve que $R^{\prime} / D$ et $R / D$ sont $(\leq 6)$-hypomorphes, et par suite d'après le lemme $2.1 R^{\prime} / D \sim R / D$.

Lemme 3.6. Si $|E-(C \cup D)| \geq 11$, alors $R^{\prime} / C \sim R / C$.

Preuve. Comme l'équivalence $D_{R / D, R^{\prime} / D}$ possède au moins deux classes, alors $C \cap D=\phi$. D'après le lemme 3.4 , on peut supposer que $C$ est une composante connexe de $R$ et que $R / C$ est un tournoi sans diamant non auto-dual, et par suite $R / C$ est fortement connexe. Posons $H=R /(C \cup D)$. Soient $(a, b) \in C \times D$. Considérons les parties $F$ de $E$ contenant $\{a, b\}$ et telles que $: R / F \sim H$ ou $R / F \sim H^{\star}$. Il n'y en a pas d'autre que $C \cup D$ car $H=R /(C \cup D)$ admet deux composantes connexes $C$ et $D$. Le nombre de parties $F$ de $E$ contenant $\{a, b\}$ telles que $R / F$ est 
hémimorphe à $H$ est donc égal à 1 (c.a.d $n(R, H,\{a, b\})=1$ ). D'après le lemme 2.7, le nombre de parties $F$ de $E$ contenant $\{a, b\}$ telles que $R^{\prime} / F$ est hémimorphe à $H$ est donc égal à 1 (c.a.d $\left.n\left(R^{\prime}, H,\{a, b\}\right)=1\right)$. Il s'ensuit que $R^{\prime} /(C \cup D) \sim H=R /(C \cup D)$ ou $R^{\prime} /(C \cup D) \sim H^{\star}$. Comme $C$ et $D$ sont les composantes connexes de $R /(C \cup D)$ et $R^{\prime} / D \sim R / D$, alors $R^{\prime} / C \sim R / C$.

Lemme 3.7. Si $|E-D| \geq 17$, alors $R / C \sim R^{\prime} / C$.

Preuve. Si $|C| \leq 6$, alors le lemme 3.6 prouve que $R / C \sim R^{\prime} / C$. Dans la suite $|C| \geq 7$. Soient $A$ une partie de $C$ de cardinal $\leq 6, b \in D$ et $H=$ $R /(A \cup D)$. Si $R / A$ est auto-dual, on a trivialement l'isomorphisme entre $R / A$ et $R^{\prime} / A$. Dans La suite, $R / A$ n'est pas auto-dual. On va distinguer les cas suivants :

$c_{1}$ ) Cas où $R / C$ et $R / D$ ne sont pas hémimorphes.

Considérons les parties $F$ de $E$ contenant $\{b\} \cup A$ et telles que $: R / F \sim$ $H$ ou $R / F \sim H^{\star}$. Il n'y en a pas d'autre que $A \cup D$, car $H=R /(A \cup D)$ admet deux composantes connexes $A$ et $D$. Le nombre de parties $F$ de $E$ contenant $\{b\} \cup A$ telles que $R / F$ est hémimorphe à $H$ est donc égal à 1 (c.a.d $n(R, H,\{b\} \cup A)=1)$. D'après le lemme 2.7, $n\left(R^{\prime}, H,\{b\} \cup A\right)=1$. Il s'ensuit que $R^{\prime} /(A \cup D) \sim H$ ou $R^{\prime} /(A \cup D) \sim H^{\star}$. Comme $A$ et $D$ sont les composantes connexes de $R /(A \cup D)$ et $R^{\prime} / D \sim R / D$, alors $R^{\prime} / A \sim R / A$.

$\left.c_{2}\right)$ Cas $R / C$ et $R / D$ sont hémimorphes.

Soit $x \in(C-A)$. Considérons alors les parties $F$ de $E-\{x\}$ contenant $\{b\} \cup A$ et telles que : $R / F \sim H$ ou $R / F \sim H^{\star}$. Il n'y en a pas d'autre que $A \cup D$ car $A$ et $D$ sont les composantes connexes de $H=R /(A \cup D)$. Le nombre de parties $F$ de $E-\{x\}$ contenant $\{b\} \cup A$ telles que R/F est hémimorphe à $H$ est donc égal à 1 (c.a.d $n(R /(E-\{x\}), H,\{b\} \cup A)=1$ ) . D'après le lemme 2.7, $\left.n\left(R^{\prime} /(E-\{x\}), H,\{b\} \cup A\right)=1\right)$. Il s'ensuit que $R^{\prime} /(A \cup D) \sim H$ ou $R^{\prime} /(A \cup D) \sim H^{\star}$. Comme $A$ et $D$ sont les composantes connexes de $H=R /(A \cup D)$ et $R^{\prime} / D \sim R / D$, alors $R^{\prime} / A \sim R / A$.

Dans les deux cas $R^{\prime} / A \sim R / A$. Il s'ensuit que $R / C$ et $R^{\prime} / C$ sont ( $\leq 6)$-hypomorphes, et par suite d'après le lemme $2.1 R / C \sim R^{\prime} / C$.

Lemme 3.8. Si $h \geq 1$ et si $|D| \geq 6+h$, alors il existe une partie $A$ de $D$ de cardinal $h$ tel que $R /(D-A)$ et $R^{\prime} /(D-A)$ sont isomorphes mais non anti-isomorphes.

Preuve. Soit $h \geq 1$. Si pour toute partie $A$ de $D$ de cardinal $h$, $R^{\star} /(D-A) \sim R /(D-A)$, alors $R^{\star} / D$ et $R / D$ sont (-h)-hypomorphes 
et comme $R / D$ et $R^{\star} / D$ sont $(\leq 4)$-hypomorphes et $|D| \geq 6+h$, alors $R^{\star} / D \sim R / D$ (lemme 2.8), ce qui est impossible. D'ou il existe $A \subset D$ tel que $R /(E-A)$ est non auto-dual. D'après le lemme $3.5 R / D$ et $R^{\prime} / D$ sont ( $\leq 6)$-hypomorphes, alors $R /(D-A)$ et $R^{\prime} /(D-A)$ sont aussi $(\leq 6)$ hypomorphes, et par suite $R^{\prime} /(D-A) \sim R /(D-A)$ (lemme 2.1). Il s'ensuit que $R /(D-A)$ et $R^{\prime} /(D-A)$ sont isomorphes mais non anti-isomorphes.

Lemme 3.9. $R / C \sim R^{\prime} / C$.

Preuve. D'après le lemme $3.4 C$ et $D$ sont deux composantes connexes de $R$ qui sont deux tournois sans diamant. Posons $X=E-(C \cup D)$. D'après le lemme 3.6 on peut supposer que $|X| \leq 10$, de même d'après le lemme 3.7 on suppose que $|E-D| \leq 16$. Suivant le cardinal de $C$, on a :

Cas $1 \mathrm{Si}|C| \leq 6$.

Suivant le cardinal de $X$, on a :

$\left.c_{1}\right)|X|=10$. D'après le lemme 3.8 il existe une partie $A$ de $D$ de cardinal 1 tel que $R /(D-A)$ et $R^{\prime} /(D-A)$ sont isomorphes mais non antiisomorphes. Soit $f$ un hémimorphisme de $R /(E-A \cup X)$ sur $R^{\prime} /(E-A \cup X)$. Si $f$ est un isomorphisme, comme $R^{\prime} /(D-A) \sim R /(D-A)$, alors le lemme 2.6 prouve que $R / C \sim R^{\prime} / C$. Si $f$ est un anti-isomorphisme. Comme $C$ est connexe, alors d'après le lemme $2.3 R^{\prime} / C \sim R / C$ ou $R^{\prime} / C \sim R^{\star} / C$. Si $R^{\prime} / C \sim R^{\star} / C$, alors d'après le lemme $2.6 R^{\prime} /(D-A) \sim R^{\star} /(D-A)$, ce qui est impossible. Donc $R / C \sim R^{\prime} / C$.

$\left.c_{2}\right)|X| \leq 9$. D'après le lemme 3.8 il existe une partie $A$ de $D$ tel que $|X|+|A|=11$ et $\left(R /(D-A)\right.$ et $\left.R^{\prime} /(D-A)\right)$ sont isomorphes mais non anti-isomorphes. Nous montrons comme dans le cas $c_{1}$ que $R / C \sim R^{\prime} / C$.

Cas 2 Si $|C| \geq 7$.

Soient $J$ une partie de $C$ de cardinal $h$ tel que $|C|-h=6$. D'après le lemme 3.8 il existe une partie $A$ de $D$ telle que $|A|+|J|+|X|=11$ et $R /(D-A)$ et $R^{\prime} /(D-A)$ sont isomorphes mais non anti-isomorphes. Soit $f$ un hémimorphisme de $R /(E-A \cup X \cup J)$ sur $R^{\prime} /(E-A \cup X \cup J)$.

- Si $f$ est un isomorphisme. Comme $R^{\prime} /(D-A) \sim R /(D-A)$, alors le lemme 2.6 prouve que $R^{\prime} /(C-J) \sim R /(C-J)$.

- Si $f$ est un anti-isomorphisme. Comme $|(C-J)|=6$, alors $R^{\prime} /(C-$ $J) \sim R /(C-J)$ ou $R^{\prime} /(C-J) \sim R^{\star} /(C-J)$. Si $R^{\prime} /(C-J) \sim R^{\star} /(C-J)$, alors d'après le lemme $2.6 R^{\prime} /(D-A) \sim R^{\star} /(D-A)$ ce qui est impossible. Donc $R^{\prime} /(C-J) \sim R /(C-J)$.

Dans les deux cas $R^{\prime} /(C-J) \sim R /(C-J)$. Les restrictions $R / C$ et $R^{\prime} / C$ sont donc $(4,-h)$-hypomorphes et $|C|-h=6$. Il s'ensuit que 
$R / C \sim R^{\prime} / C$ (lemme 2.8).

\section{Optimalité de la valeur $23 \mathrm{du}$ théorème 3.1.}

Dans ce paragraphe, nous donnons un contre-exemple, prouvant l'optimalité de la valeur 23 du théorème 3.1 . On considère : La relation $R_{1}=C_{3}\left(T_{1}, T_{2}, T_{3}\right)$ où $C_{3}$ est un 3 -cycle de base $\{1,2,3\}$ et pour tout $i, T_{i}$ est une $i$-chaine. Avec ces notations on peut voir que les relations :

$R=S\left(R_{1}, R_{1}, T_{10}\right)$ et $R^{\prime}=S\left(R_{1}, R^{\star}{ }_{1}, T_{10}\right)$ (où $S$ est une relation vide à 3 éléments) sont (-11)-hémimorphes, sans être hémimorphes sur un ensemble de cardinal 22 .

\section{La (-10)-demi-reconstructibilité des relations binaires finies}

Théorème 4.1. . Les relations binaires finies de cardinal $n \geq 22$ sont $(n-10)$-demi-reconstructibles.

Soient $R$ et $R^{\prime}$ deux relations binaires $(n-10)$-hémimorphes sur une base finie $E$ de cardinal $n$ au moins égal à 22 et $k$ le plus petit cardinal des restrictions non auto-duales de $R$. Le lemme 2.5 prouve que les relations $R$ et $R^{\prime}$ sont $(\leq 10)$-hémimorphes. On a :

-Si $R$ est connexe, alors le lemme 2.2 prouve que $R$ est $(n-10)$-demireconstructible.

- Si $2 \leq k \leq 4$, alors le lemme 2.4 prouve que $R$ est 10 -demi-reconstructible et par suite $R$ est $(n-10)$-demi-reconstructible (lemme 2.5 ).

- Si $k \geq 7$ ou $k=0$. Dans ce cas $R$ n'admet aucune restriction non autoduale de cardinal inférieur au égal à 6 , alors le lemme 2.1 prouve que $R$ est $(n-10)$-demi-reconstructible.

Dans la suite $k \in\{5,6\}$ donc les relations $R, R^{\star}$ et $R^{\prime}$ sont deux à deux $(\leq 4)$-hypomorphes. Soit $D$ la plus grande composante connexe non auto-duale de $R$. D'après le $i$ ) du corollaire $2.16 R / D$ est un tournoi sans diamant ou un élément de $E\left(S_{n}\right)$, de même le lemme 2.13 prouve que l'une des équivalences $D_{R / D, R^{\prime} / D}$ ou $D_{R^{\star} / D, R^{\prime} / D}$ possède au moins deux classes.

La preuve du théorème 3.1 utilise les résultats suivants :

Proposition 4.2. Soient $D$ la plus grande composante connexe non autoduale de $R$. Si l'équivalence $D_{R / D, R^{\prime} / D}$ possède au moins deux classes, alors $R^{\prime} \sim R$. 
Notons que comme conséquence de la proposition 4.2 on a :

Corollaire 4.3. Soient $D$ la plus grande composante connexe non autoduale de $R$. Si l'équivalence $D_{R^{\star} / D, R^{\prime} / D}$ possède au moins deux classes, alors $R^{\prime} \sim R^{\star}$.

Dans la suite de la preuve de la proposition 4.2C est une classe de l'équivalence $D_{R, R^{\prime}}$. Comme $k \in\{5,6\}$, alors les relations $R$ et $R^{\prime}$ sont $(\leq 4)$-hypomorphes, et par suite d'après le lemme $2.9 C$ est un intervalle de $R$ et $R^{\prime}$, sur lequel les restrictions de $R$ et $R^{\prime}$ sont réflexives ou irréflexives, la preuve de la proposition 4.2 utilise les lemmes suivants :

Lemme 4.4. i) Si $C$ est différente de sa composante connexe, alors $R^{\prime} / C \sim$ $R / C$.

ii) Si $R / C$ n'est ni un tournoi sans diamant ni un élément de $E\left(S_{n}\right)$, alors $R^{\prime} / C \sim R / C$.

iii) Si $R / C$ est auto-dual, alors $R^{\prime} / C \sim R / C$.

Preuve. i)Si $C$ est différente de sa composante connexe, alors le lemme 2.12 prouve que $R^{\prime} / C \sim R / C$.

ii) On suppose que $C$ est une composante connexe de $R$. Comme $k \in$ $\{5,6\}$ et $R / C$ n'est ni un tournoi sans diamant ni un élément de $E\left(S_{n}\right)$, alors d'après le lemme $2.10 R^{\prime} / C \sim R / C$.

iii) Comme $R / C$ est connexe, alors le lemme 2.3 prouve que $R^{\prime} / C \sim$ $R / C$ ou $R^{\prime} / C \sim R^{\star} / C$. Si $R / C$ est auto-dual, alors $R^{\prime} / C \sim R / C \sim R^{\star} / C$.

Dans la suite on suppose que $C$ et $D$ sont deux composantes connexes non auto-duales de $R$ qui sont des tournois sans diamant ou des éléments de $E\left(S_{n}\right)$.

Lemme 4.5. Si $|E-(C \cup D)| \geq 10$, alors $R^{\prime} / C \sim R / C$.

Preuve. La preuve est analogue à la preuve du lemme 3.6.

Lemme 4.6. Si $|E-D| \geq 16$, alors $R / C \sim R^{\prime} / C$.

Preuve. La preuve est analogue à la preuve du lemme 3.7.

Lemme 4.7. $R / C \sim R^{\prime} / C$. 
Preuve. La preuve est analogue à la preuve du lemme 3.9.

\section{Optimalité de la valeur $22 \mathrm{du}$ théorème 4.1 .}

Dans ce paragraphe, nous donnons un contre-exemple, prouvant l'optimalité de la valeur 22 du théorème 4.1. On considère : La relation $R_{1}=C_{3}\left(T_{1}, T_{2}, T_{3}\right)$ où $\mathrm{C} 3$ est un 3 -cycle de base $\{1,2,3\}$ et pour tout $i, T_{i}$ est une $i$-chaine. Avec ces notations on peut voir que les relations :

$R=S\left(R_{1}, R_{1}, T_{9}\right)$ et $R^{\prime}=S\left(R_{1}, R^{\star}{ }_{1}, T_{9}\right)$ (S est une relation vide a 3 éléments) sont (-10)-hémimorphes, sans être hémimorphes sur un ensemble de cardinal 21.

\section{La (-9)-demi-reconstructibilité des relations binaires finies.}

Théorème 5.1. Les relations binaires finies de cardinal $n \geq 21$ sont ( $n-$ 9)-demi-reconstructibles.

Soient $R$ et $R^{\prime}$ deux relations binaires $(n-9)$-hémimorphes sur une base finie $E$ de cardinal $n$ au moins égal à 21 et $k$ le plus petit cardinal des restrictions non auto-duales de $R$, et $I_{0}$ la base d'une de ces restrictions. Le lemme 2.5 prouve que les relations $R$ et $R^{\prime}$ sont $(\leq 9)$-hémimorphes. On a :

- Si $R$ est connexe, alors le lemme 2.2 prouve que $R$ est $(n-9)$-demireconstructible.

- Si $2 \leq k \leq 3$, alors le lemme 2.4 prouve que $R$ est 9 -demi-reconstructible et par suite $R$ est $(n-9)$-demi-reconstructible (lemme 2.5).

- Si $k \geq 7$ ou $k=0$. Dans ce cas $R$ n'admet aucune restriction non auto-duale de cardinal inférieur ou égal à 6 , alors le lemme 2.1 prouve que $\mathrm{R}$ est $(n-9)$-demi-reconstructible.

- Si $k \in\{5,6\}$, alors les relations $R, R^{\star}$ et $R^{\prime}$ sont deux à deux $(\leq 4)$ hypomorphes. Soient $D$ la plus grande composante connexe non auto-duale de $R$, nous montrons comme dans la proposition 3.2 que si l'équivalence $D_{R / D, R^{\prime} / D}\left(\right.$ resp. $\left.D_{R^{\star} / D, R^{\prime} / D}\right)$ possède au moins deux classes, alors $R^{\prime} \sim R$ (resp. $R^{\prime} \sim R^{\star}$ ).

Dans la suite $k=4$, donc les relations $R, R^{\star}$ et $R^{\prime}$ sont deux à deux $(\leq 3)$-hypomorphes, et par suite d'après le lemme 2.9 toute classe $C$ de l'équivalence $D_{R, R^{\prime}}$ est un intervalle de $R$ et $R^{\prime}$, sur lequel les restrictions de $R$ et $R^{\prime}$ sont réflexives ou irréflexives.

La preuve du théorème 5.1 utilise les résultats suivants : 
Proposition 5.2. Si $R^{\prime} / I_{0} \sim R / I_{0}$, alors $R^{\prime} \sim R$.

Notons que comme conséquence de la proposition 5.2 on a :

Corollaire 5.3. Si $R^{\prime} / I_{0} \sim R^{\star} / I_{0}$, alors $R^{\prime} \sim R^{\star}$.

Dans la suite de la preuve de la proposition 5.2, $C$ est une classe de l'équivalence $D_{R, R^{\prime}}$, la preuve utilise les lemmes suivants :

Lemme 5.4. i) Si $C$ est différente de sa composante connexe, alors $R^{\prime} / C \sim$ $R / C$.

ii) Si $R / C$ est auto-dual, alors $R^{\prime} / C \sim R / C$.

Preuve. i) Si $C$ est différente de sa composante connexe, alors le lemme 2.12 prouve que $R^{\prime} / C \sim R / C$.

ii)Comme $R / C$ est connexe, alors le lemme 2.3 prouve que $R^{\prime} / C \sim R / C$ ou $R^{\prime} / C \sim R^{\star} / C$. Si $R / C$ est auto-dual, alors $R^{\prime} / C \sim R / C \sim R^{\star} / C$.

Dans la suite on suppose $C$ est une composante connexe non auto-duale de $R$.

Lemme 5.5. $I_{0} \cap C=\phi$.

Preuve. Puisque $R / I_{0}$ n'est pas un drapeau, et $R^{\prime} / I_{0} \sim R / I_{0}$, alors d'après le i) du lemme $2.21 I_{0} \cap C=\phi$.

Lemme 5.6. i) $R / C$ et $R^{\prime} / C$ sont $(\leq 5)$-hypomorphes.

ii) Si $R / C$ n'est pas un tournoi sans diamant, alors $R^{\prime} / C \sim R / C$.

Preuve. i) Comme $R$ et $R^{\prime}$ sont $(\leq 9)$-hémimorphes et $R^{\prime} / I_{0} \sim R / I_{0}$, et $\left|I_{0}\right|=4$, alors le lemme 2.13 prouve que $R^{\prime} / C$ et $R / C$ sont $(\leq 5)$ hypomorphes.

ii) Puisque $R^{\prime} / C$ et $R / C$ sont $(\leq 5)$-hypomorphes, alors le lemme 2.10 de G. Lopez et C. Rauzy nous donne la forme de $R / C$ et de $R^{\prime} / C$, d'après la forme si $R / C$ n'est ni un tournoi sans diamant ni un élément de $E\left(S_{n}\right)$, alors $R^{\prime} / C \sim R / C$. Dans le cas où $R / C$ est un élément de $E\left(S_{n}\right)$, le lemme 2.14 prouve que $R^{\prime} / C \sim R / C$. Il s'ensuit que si $R / C$ n'est pas un tournoi sans diamant, alors $R^{\prime} / C \sim R / C$.

Lemme 5.7. Si $\left|E-\left(C \cup I_{0}\right)\right| \geq 9$, alors $R^{\prime} / C \sim R / C$. 
Preuve. D'après le lemme 5.4 on suppose que $C$ est une composante connexe non auto-duale de $R$, de même d'après le lemme 5.6 on suppose que $R / C$ est un tournoi sans diamant, et par suite $R / C$ est fortement connexe. Soient $H=R /\left(I_{0} \cup C\right)$, et $a \in C$. Considérons alors les parties $F$ de $E$ contenant $I_{0} \cup\{a\}$ et telles que : $R / F \sim H$ ou $R / F \sim H^{\star}$. Il n'y en a pas d'autre que $I_{0} \cup C$ car $I_{0}$ et $C$ sont les composantes connexes de $H=R /\left(I_{0} \cup C\right)$. Le nombre de parties $F$ de $E$ contenant $I_{0} \cup\{a\}$ telles que $R / F$ est hémimorphe à $H$ est donc égal à 1 (c.a.d $n\left(R, H, I_{0} \cup\{a\}\right)=1$ ). D'après le lemme 2.7, le nombre de parties $F$ de $E$ contenant $I_{0} \cup\{a\}$ telles que $R^{\prime} / F$ est hémimorphe à $H$ est donc égal à 1 (c.a.d $n\left(R^{\prime}, H, I_{0} \cup\right.$ $\{a\})=1)$. Il s'ensuit que $R^{\prime} /\left(C \cup I_{0}\right) \sim H$ ou $R^{\prime} /\left(C \cup I_{0}\right) \sim H^{\star}$. Comme $R /\left(C \cup I_{0}\right)$ admet deux composantes connexes $I_{0}$ et $C$, et $R^{\prime} / I_{0} \sim R / I_{0}$, alors $R / C \sim R^{\prime} / C$.

Lemme 5.8. $R^{\prime} / C \sim R / C$.

Preuve. D'après le lemme 2.17, on peut supposer que $R / C$ est un tournoi sans diamant. Posons $X=E-\left(C \cup I_{0}\right)$, suivant le cardinal de $x$, on les cas suivants :

- $c_{1}$ Si $|X| \geq 9$, alors le lemme 3.4 prouve que $R / C \sim R^{\prime} / C$.

- $c_{2}$ Si $|X|=8$. Soit $J$ une partie de $C$ de cardinal 1 . Comme $R / C$ est un tournoi sans diamant, alors $R /(C-J)$ est soit fortement connexe, soit une chaîne. Si $R /(C-J)$ est une chaîne, alors $R^{\prime} /(C-J) \sim R /(C-J)$. Dans la suite $R /(C-J)$ est un tournoi sans diamant fortement connexe. Soit $f$ un hémimorphisme de $R /(E-X \cup J)$ sur $R^{\prime} /(E-X \cup J)$. Comme $R /(E-X \cup J)$ admet deux composantes connexes $I_{0}$ et $C-J$ et $R^{\prime} / I_{0} \sim$ $R / I_{0}$, alors $f$ est un isomorphisme avec $f\left(I_{0}\right)=I_{0}$ et $f(C-J)=C-J$. Il s'ensuit que $R^{\prime} /(C-J) \sim R /(C-J)$. Donc $R / C$ et $R^{\prime} / C$ sont $(4,-1)$ hypomorphes et $|C| \geq 7$, et par suite d'après le lemme $2.8 R / C \sim R^{\prime} / C$.

- $c_{3}$ Si $|X|=7$. Soit $J$ une partie de $C$ de cardinal 2. Nous montrons comme dans le cas $c_{2}$ que $R^{\prime} /(C-J) \sim R /(C-J)$. Donc $R / C$ et $R^{\prime} / C$ sont $(4,-2)$-hypomorphes et $|C| \geq 8$, et par suite d'après le lemme 2.8 $R^{\prime} / C \sim R / C$.

- $c_{4} \mathrm{Si}|X| \leq 6$. Soit $J$ une partie de $C$ de cardinal $h$ tel que $h+|X|=9$. Nous montrons comme dans le cas $c_{2}$ que $R^{\prime} /(C-J) \sim R /(C-J)$. Donc $R / C$ et $R^{\prime} / C$ sont $(4,-h)$-hypomorphes et $|C| \geq 6+h$, et par suite d'après le lemme $2.8 R^{\prime} / C \sim R / C$.

\section{Optimalité de la valeur 21 du théorème 5.1.}


Dans ce paragraphe, nous donnons un contre-exemple, prouvant l'optimalité de la valeur $21 \mathrm{du}$ théorème 5.1. On considère : La relation $R_{1}=C_{3}\left(T_{1}, T_{2}, T_{3}\right)$ où $C_{3}$ est un 3 -cycle de base $\{1,2,3\}$ et pour tout $i, T_{i}$ est une $i$-chaîne. Avec ces notations on peut voir que les relations :

$R=S\left(R_{1}, R_{1}, T_{8}\right)$ et $R^{\prime}=S\left(R_{1}, R^{\star}{ }_{1}, T_{8}\right)$ (où $S$ est une relation vide à 3 éléments) sont $(-9)$-hémimorphes, sans être hémimorphes sur un ensemble de cardinal 20.

\section{La (-8)-demi-reconstructibilité des relations binaires finies.}

Théorème 6.1. Les relations binaires finies de cardinal $n \geq 20$ sont ( $n-$ 8)-demi-reconstructibles.

Soient $R$ et $R^{\prime}$ deux relations binaires $(n-8)$-hémimorphes sur une base finie $E$ de cardinal $n$ au moins égal à 20 et $k$ le plus petit cardinal des restrictions non auto-duales de $R$, et $I_{0}$ la base d'une de ces restrictions. Le lemme 2.5 prouve que les relations $R$ et $R^{\prime}$ sont $(\leq 8)$-hémimorphes. On a

-Si $R$ est connexe, alors le lemme 2.2 prouve que $R$ est $(n-8)$-demireconstructible.

- Si $k=2$, alors le lemme 2.4 prouve que $R$ est 8 -demi-reconstructible et par suite $R$ est $(n-8)$-demi-reconstructible (lemme 2.5 ).

- Si $k \geq 7$ ou $k=0$. Dans ce cas $R$ n'admet aucune restriction non auto-duale de cardinal inférieur au égal à 6 , alors le lemme 2.1 prouve que $R$ est $(n-8)$-demi-reconstructible.

- Si $k \in\{5,6\}$, alors les relations $R, R^{\star}$ et $R^{\prime}$ sont deux à deux $(\leq 4)$ hypomorphes. Soient $D$ la plus grande composante connexe non auto-duale de $R$, nous montrons comme dans la proposition 3.2 que si l'équivalence $D_{R / D, R^{\prime} / D}\left(\right.$ resp. $\left.D_{R^{\star} / D, R^{\prime} / D}\right)$ possède au moins deux classes, alors $R^{\prime} \sim R$ (resp. $R^{\prime} \sim R^{\star}$ ).

- Si $k=4$. Nous montrons comme dans la proposition 5.2 que si $R^{\prime} / I_{0} \sim$ $R / I_{0}$ (resp. $\left.R^{\prime} / I_{0} \sim R^{\star} / I_{0}\right)$, alors $R^{\prime} \sim R$ (resp. $\left.R^{\prime} \sim R^{\star}\right)$.

Dans la suite $k=3$.

La preuve du théorème 6.1 utilise la proposition suivante :

Proposition 6.2. Si $R^{\prime} / I_{0} \sim R / I_{0}$, alors $R \sim R^{\prime}$.

Notons que comme conséquence de la proposition 6.2 on a : 
Corollaire 6.3. Si $R^{\prime} / I_{0} \sim R^{\star} / I_{0}$, alors $R^{\prime} \sim R^{\star}$.

Preuve de la proposition 6.2 Soit $C$ une classe de l'équivalence $D_{R, R^{\prime}}$. Comme $R^{\prime} / I_{0} \sim R / I_{0}$, alors d'après le lemme $2.21, I_{0} \cap C=\phi$ ou $I_{0} \cap C=\{c\}$ avec $R / I_{0}$ est un drapeau, d'arête neutre $\{c, a\}$ et $\{c, b\}$.

i) Si $I_{0} \cap C=\phi$, nous montrons comme dans la proposition 5.2 que si $R^{\prime} / I_{0} \sim R / I_{0}$, alors $R / C \sim R^{\prime} / C$.

ii) Si $R / I_{0}$ est un drapeau, d'arête neutre $\{c, a\}$ et $\{c, b\}$ et $I_{0} \cap C=\{c\}$, alors le ii) du lemme 2.21 prouve que $R / C$ et $R^{\prime} / C$ sont $(\leq 6)$-hypomorphes, et par suite $R^{\prime} / C \sim R / C$ (d'après le lemme 2.1).

Optimalité de la valeur $20 \mathrm{du}$ théorème 6.1.

Dans ce paragraphe, nous donnons un contre-exemple, prouvant l'optimalité de la valeur 20 du théorème 6.1. On considère : La relation $R_{1}=C_{3}\left(T_{1}, T_{2}, T_{3}\right)$ où $C_{3}$ est un 3 -cycle de base $\{1,2,3\}$ et pour tout $i, T_{i}$ est une $i$-chaîne. Avec ces notations on peut voir que les relations :

$R=S\left(R_{1}, R_{1}, T_{7}\right)$ et $R^{\prime}=S\left(R_{1}, R^{\star}{ }_{1}, T_{7}\right)$ (où $S$ est une relation vide à 3 éléments) sont (-8)-hémimorphes, sans être hémimorphes sur un ensemble de cardinal 19.

\section{La (-7)-demi-reconstructibilité des relations binaires finies.}

Théorème 7.1. Les relations binaires finies de cardinal $n \geq 25$ sont ( $n-$ 7)-demi-reconstructibles.

Soient $R$ et $R^{\prime}$ deux relations binaires $(n-7)$-hémimorphes sur une base finie $E$ de cardinal n au moins égal à 25 et $k$ le plus petit cardinal des restrictions non auto-duales de $R$, et $I_{0}$ la base d'une de ces restrictions. Le lemme 2.5 prouve que les relations $R$ et $R^{\prime}$ sont $(\leq 7)$-hémimorphes. On a :

- Si $R$ est connexe, alors le lemme 2.2 prouve que $R$ est $(n-7)$-demireconstructible.

- Si $k \geq 7$ ou $k=0$. Dans ce cas $R$ n'admet aucune restriction non auto-duale de cardinal inférieur ou égal à 6 , alors le lemme 2.1 prouve que $\mathrm{R}$ est $(n-7)$-demi-reconstructible.

- Si $k \in\{5,6\}$, alors les relations $R, R^{\star}$ et $R^{\prime}$ sont deux à deux $(\leq 4)$ hypomorphes. Soient $D$ la plus grande composante connexe non auto-duale de $R$, nous montrons comme dans la proposition 3.2 que si l'équivalence $D_{R / D, R^{\prime} / D}$ (resp. $\left.D_{R^{\star} / D, R^{\prime} / D}\right)$ possède au moins deux classes, alors $R^{\prime} \sim R$ (resp. $R^{\prime} \sim R^{\star}$ ). 
- Si $k \in\{2,3\}$. Nous montrons comme dans la proposition 5.2 que si $R^{\prime} / I_{0} \sim R / I_{0}$ (resp. $\left.R^{\prime} / I_{0} \sim R^{\star} / I_{0}\right)$, alors $R^{\prime} \sim R\left(\right.$ resp. $\left.R^{\prime} \sim R^{\star}\right)$.

Dans la suite $k=4$, donc les relations $R, R^{\star}$ et $R^{\prime}$ sont deux à deux $(\leq 3)$-hypomorphes, et par suite d'après le lemme 2.9 toute classe $C$ de l'équivalence $D_{R, R^{\prime}}$ est un intervalle de $R$ et $R^{\prime}$, sur lequel les restrictions de $R$ et $R^{\prime}$ sont réflexives ou irréflexives.

La preuve du théorème 7.1 se base sur la proposition suivante :

Proposition 7.2. Soient $D$ la plus grande composante connexe non autoduale de $R$. Si l'équivalence $D_{R / D, R^{\prime} / D}$ possède au moins deux classes, alors $R^{\prime} \sim R$.

Notons que comme conséquence de la proposition 7.2 on a :

Corollaire 7.3. Soient $D$ la plus grande composante connexe non autoduale de $R$. Si l'équivalence $D_{R^{\star} / D, R^{\prime} / D}$ possède au moins deux classes, alors $R^{\prime} \sim R^{\star}$.

Dans la suite de la preuve de la proposition 7.2, $C$ est une classe de l'équivalence $D_{R, R^{\prime}}$, la preuve utilise les lemmes suivants :

Lemme 7.4. i) Si $C$ est différente de sa composante connexe, alors $R^{\prime} / C \sim$ $R / C$.

ii) Si $R / C$ est auto-dual, alors $R^{\prime} / C \sim R / C$.

Preuve. $\quad$ i) Si $C$ est différente de sa composante connexe, alors le lemme 2.12 prouve que $R^{\prime} / C \sim R / C$.

ii) Comme $R / C$ est connexe, alors le lemme 2.3 prouve que $R^{\prime} / C \sim$ $R / C$ ou $R^{\prime} / C \sim R^{\star} / C$. Si $R / C$ est auto-dual, alors $R^{\prime} / C \sim R / C \sim R^{\star} / C$.

Dans la suite $\mathrm{C}$ est une composante connexe non auto-duale de $R$.

Lemme 7.5. $S i|E-(C \cup D)| \geq 7$, alors $R / C \sim R^{\prime} / C$.

Preuve. La preuve est analogue à la preuve du lemme 3.6.

Lemme 7.6. Si $|E-D| \geq 13$, alors $R / C \sim R^{\prime} / C$. 
Preuve. La preuve est analogue à la preuve du lemme 3.7.

Lemme 7.7. Si $h \geq 4$ et si $|D| \geq 6+h$, alors il existe une partie $A$ de $D$ de cardinal $h$ tel que $R /(D-A)$ et $R^{\prime} /(D-A)$ sont isomorphes mais non anti-isomorphes.

Preuve. Soit $h \geq 4$. Si pour toute partie $A$ de $D$ de cardinal $h$, $R^{\star} /(D-A) \sim R /(D-A)$, alors $R^{\star} / D$ et $R / D$ sont $(-h)$-hypomorphes et $|D| \geq 6+h$, alors $R^{\star} / D \sim R / D$ (lemme 2.8), ce qui est impossible. D'ou il existe $A \subset D$ telle que $R /(E-A)$ est non auto-dual. D'après le $i)$ du lemme $2.22 R / D$ et $R^{\prime} / D$ sont $(\leq 6)$-hypomorphes, alors $R /(D-A)$ et $R^{\prime} /(D-A)$ sont aussi $(\leq 6)$-hypomorphes, et par suite $R^{\prime} /(D-A) \sim R /(D-A)$ (lemme 2.1). Il s'ensuit que $R /(D-A)$ et $R^{\prime} /(D-A)$ sont isomorphes mais non anti-isomorphes.

Lemme 7.8. $R / C \sim R^{\prime} / C$.

Preuve. Posons $X=E-(C \cup D)$. D'après le lemme 7.5 on peut supposer que $|X| \leq 6$, de même d'après le lemme 7.6 on suppose que $|E-D| \leq 12$, et par suite $|C| \leq 12$. Suivant le cardinal de $X$, on a :

$\left.c_{1}\right)|X|=0$. D'après le lemme 7.7 il existe une partie $A$ de $D$ de cardinal 7 telle que $R /(D-A)$ et $R^{\prime} /(D-A)$ sont isomorphes mais non anti-isomorphes. Soit $f$ un hémimorphisme de $R /(E-A)$ sur $R^{\prime} /(E-A)$.

- Si $f$ est un isomorphisme, comme $R^{\prime} /(D-A) \sim R /(D-A)$, alors le lemme 2.6 prouve que $R / C \sim R^{\prime} / C$.

- Si $f$ est un anti-isomorphisme. Comme $C$ est connexe, alors d'après le lemme $2.3 R^{\prime} / C \sim R / C$ ou $R^{\prime} / C \sim R^{\star} / C$. Si $R^{\prime} / C \sim R^{\star} / C$, alors d'après le lemme $2.6 R^{\prime} /(D-A) \sim R^{\star} /(D-A)$, ce qui est impossible. Donc $R / C \sim R^{\prime} / C$.

$\left.c_{2}\right)|X|=1$. D'après le lemme 7.7 il existe une partie $A$ de $D$ de cardinal 6 tel que $R /(D-A)$ et $R^{\prime} /(D-A)$ sont isomorphes mais non antiisomorphes. Soit $f$ un hémimorphisme de $R /(E-A \cup X) \operatorname{sur} R^{\prime} /(E-A \cup X)$.

- Si $f$ est un isomorphisme, comme $R^{\prime} /(D-A) \sim R /(D-A)$, alors le lemme 2.6 prouve que $R / C \sim R^{\prime} / C$.

- Si $f$ est un anti-isomorphisme. Comme $C$ est connexe, alors d'après le lemme $2.3 R^{\prime} / C \sim R / C$ ou $R^{\prime} / C \sim R^{\star} / C$. Si $R^{\prime} / C \sim R^{\star} / C$, alors d'après le lemme $2.6 R^{\prime} /(D-A) \sim R^{\star} /(D-A)$, ce qui est impossible. Donc $R / C \sim R^{\prime} / C$.

$\left.c_{3}\right)|X|=2$. D'après le lemme 7.7 il existe une partie $A$ de $D$ de cardinal 5 telle que $R /(D-A)$ et $R^{\prime} /(D-A)$ sont isomorphes mais non antiisomorphes. Soit $f$ un hémimorphisme de $R /(E-A \cup X)$ sur $R^{\prime} /(E-A \cup X)$. Nous montrons comme dans le cas $c_{2}$ que $R / C \sim R^{\prime} / C$. 
$\left.c_{4}\right)|X|=3$. D'après le lemme 7.7 il existe une partie $A$ de $D$ de cardinal 4 telle que $R /(D-A)$ et $R^{\prime} /(D-A)$ sont isomorphes mais non antiisomorphes. Soit $f$ un hémimorphisme de $R /(E-A \cup X)$ sur $R^{\prime} /(E-A \cup X)$. Nous montrons comme dans le cas $c_{2}$ que $R / C \sim R^{\prime} / C$.

$\left.c_{5}\right) 4 \leq|X| \leq 6$. D'après le lemme 7.7 il existe une partie $A$ de $D$ de cardinal 4 telle que $R /(D-A)$ et $R^{\prime} /(D-A)$ sont isomorphes mais non anti-isomorphes. Soient $B$ une partie de $X$ de cardinal 3 et $f$ un hémimorphisme de $R /(E-A \cup X \cup B)$ sur $R^{\prime} /(E-A \cup X \cup B)$. Nous montrons comme dans le cas $c_{2}$ que $R / C \sim R^{\prime} / C$.

\section{La (-6)-demi-reconstructibilité des relations binaires finies.}

Théorème 8.1. Les relations binaires finies de cardinal $n \geq 23$ sont $(n-$ 6)-demi-reconstructibles.

Soient $R$ et $R^{\prime}$ deux relations binaires $(n-6)$-hémimorphes sur une base finie $E$ de cardinal n au moins égal à 23 et $k$ le plus petit cardinal des restrictions non auto-duales de $R, I_{0}$ la base d'une de ces restrictions, et $C$ une classe de l'équivalence $D_{R, R^{\prime}}$. Le lemme 2.5 prouve que les relations $R$ et $R^{\prime}$ sont $(\leq 6)$-hémimorphes. On a :

-Si $R$ est connexe, alors le lemme 2.2 prouve que $R$ est $(n-6)$-demireconstructible.

- Si $k \geq 7$ ou $k=0$. Dans ce cas $R$ n'admet aucune restriction non auto-duale de cardinal inférieur au égal à 6 , et par suite le lemme 2.1 prouve que $\mathrm{R}$ est $(n-6)$-demi-reconstructible.

- Si $k \in\{5,6\}$, alors les relations $R, R^{\star}$ et $R^{\prime}$ sont deux à deux $(\leq 4)$ hypomorphes. Soient $D$ la plus grande composante connexe non auto-duale de $R$, nous montrons comme dans la proposition 3.2 que si l'équivalence $D_{R / D, R^{\prime} / D}$ (resp. $\left.D_{R^{\star} / D, R^{\prime} / D}\right)$ possède au moins deux classes, alors $R^{\prime} \sim R$ (resp. $R^{\prime} \sim R^{\star}$ ).

- Si $k \in\{2,3\}$, nous montrons comme dans la proposition 5.2 que si $R^{\prime} / I_{0} \sim R / I_{0}$ (resp. $\left.R^{\prime} / I_{0} \sim R^{\star} / I_{0}\right)$, alors $R^{\prime} \sim R$ (resp. $\left.R^{\prime} \sim R^{\star}\right)$.

Dans la suite $k=4$, donc les relations $R, R^{\star}$ et $R^{\prime}$ sont deux à deux $(\leq 3)$-hypomorphes, et par suite d'après le lemme 2.9 toute classe $C$ de l'équivalence $D_{R, R^{\prime}}$ est un intervalle de $R$ et $R^{\prime}$, sur lequel les restrictions de $R$ et $R^{\prime}$ sont réflexives ou irréflexives.

La preuve du théorème 8.1 se base sur la proposition suivante : 
Proposition 8.2. Soient $C$ une classe de l'équivalence $D_{R, R^{\prime}}$, et $D$ la plus grande composante connexe non auto-duale de $R$. Si l'équivalence $D_{R / D, R^{\prime} / D}$ possède au moins deux classes, alors $R^{\prime} / C \sim R / C$.

Notons que comme conséquence de la proposition 8.2 on a :

Corollaire 8.3. Soient $D$ la plus grande composante connexe non autoduale de $R$. Si l'équivalence $D_{R^{\star} / D, R^{\prime} / D}$ possède au moins deux classes, alors $R^{\prime} \sim R^{\star}$.

Dans la suite de la preuve de la proposition 8.2, $C$ est une classe de l'équivalence $D_{R, R^{\prime}}$, la preuve utilise les lemmes suivants :

Lemme 8.4. $S i|E-C| \geq 6$, alors $R / C \sim R^{\prime} / C$ ou $R^{\prime} / C \sim R^{\star} / C$.

Preuve. d'après le lemme 2.12 on suppose que $C$ est une composante connexe de $R$. Soient $H=R / C$, et $a \in C$. Considérons alors les parties $F$ de $E$ contenant $a$ et telles que $: R / F \sim H$ ou $R / F \sim H^{\star}$. Comme $C$ est une composante connexe de $R$, alors il n'y en a pas d'autre que $C$. Le nombre de parties $F$ de $E$ contenant $a$ telle que $R / F$ est hémimorphe à $H$ est donc égal à 1 (c.a.d $n(R, H,\{a\})=1$ ). D'après le lemme 2.7 , le nombre de parties $F$ de $E$ contenant $a$ telles que $R^{\prime} / F$ est hémimorphe à $H$ est donc égal à 1 (c.a.d $n\left(R^{\prime}, H,\{a\}\right)=1$ ). Il s'ensuit que $R / C \sim R^{\prime} / C$ ou $R^{\prime} / C \sim R^{\star} / C$.

Lemme 8.5. Si $|E-C \cup D| \geq 7$, alors $R^{\prime} / C \sim R / C$.

Preuve. La preuve est analogue à la preuve du lemme 3.6.

Lemme 8.6. Si $|E-D| \geq 12$, alors $R / C \sim R^{\prime} / C$.

Preuve. La preuve est analogue à la preuve du lemme 3.7.

Dans la suite $|E-D| \leq 11$, et par suite $|C| \leq 11$.

Lemme 8.7. $i$ ) Si l'équivalence $D_{R / D, R^{\prime} / D}$ possède une classe fortement connexe de cardinal $\geq 10$, alors $R / C \sim R^{\prime} / C$.

ii) Si toute classe fortement connexe de l'équivalence $D_{R / D, R^{\prime} / D}$ est de cardinal $\leq 9$, alors $R / D$ et $R^{\prime} / D$ sont $(\leq 6)$-hypomorphes. 
Preuve. Soit $K$ une classe de l'équivalence $D_{R / D, R^{\prime} / D}$. Puisque $K$ est différente de $D$, alors il existe $x \in D-K$ tel que $R(x, K) \neq R(K, x)$.

Montrons que $K$ est un tournoi sans diamant.

On va raisonner par l'absurde supposons qu'il existe une arête neutre $\{a, b\}$ de $K$. D'ou $R /\{x, a, b\}$ est un pic, ce qui est impossible, donc $K$ est un tournoi. Comme $K \neq D$, alors le i) du lemme 2.22 prouve que $R / K$ et $R^{\prime} / K$ sont ( $\left.\leq 5\right)$-hypomorphes, et par suite d'après le lemme $2.10 R / K$ est un tournoi sans diamant.

i) D'après le lemme 2.12 on suppose que $C$ est une composante connexe de $R$ et par suite $C \cap D=\phi$, de même d'après le lemme 8.6 on suppose que $|C| \leq 11$. Soient $H=R /(C \cup K \cup\{x\})$ et $Y=E-H$. On va distinguer les cas suivants :

$\left.c_{1}\right)$ Si $|Y| \geq 6$.

Soient $a$ un point de $C$ et $b, c, d$ trois points de $K$, avec $R /\{b, c, d\}$ est un 3 -cycle. Considérons alors les parties $F$ de $E$ contenant $\{x, a, b, c, d\}$ et telles que : $R / F \sim H$ ou $R / F \sim H^{\star}$. Il n'y en a pas d'autre que $C \cup K \cup\{x\}$, car $C$ et $K \cup\{x\}$ sont les composantes connexes de $H=$ $R /(C \cup K \cup\{x\})$ et $|C| \leq|K \cup\{x\}|$. Le nombre de parties $F$ de $E$ contenant $\{x, a, b, c, d\}$ telles que $R / F$ est hémimorphe à $H$ est donc égal à 1 (c.a.d $n(R, H,\{x, a, b, c, d\})=1$ ). D'après le lemme 2.7, le nombre de parties $F$ de $E$ contenant $\{x, a, b, c, d\}$ telle que $R^{\prime} / F$ est hémimorphe à $H$ est donc égal à 1 (c.a.d $\left.n\left(R^{\prime}, H,\{x, a, b, c, d\}\right)=1\right)$. Il s'ensuit que $R / C \sim R^{\prime} / C$.

$\left.c_{2}\right) \mathrm{Si}|Y|=5$.

Soient $J$ une partie de $K$ de cardinal 1 tel que $R /(K-J)$ est un tournoi sans diamant fortement connexe. Comme $C$ et $\{x\} \cup K-J$ sont les composantes connexes de $R /(E-Y \cup J)$, et $R /(\{x\} \cup K-J) \sim R^{\prime} /(\{x\} \cup$ $K-J)$, alors $R^{\prime} /(E-Y \cup J) \sim R /(E-Y \cup J)$, et par suite $R / C \sim R^{\prime} / C$.

$\left.c_{3}\right)$ Si $|Y|=4$.

Soient $J$ une partie de $K$ de cardinal 2 tel que $R /(K-J)$ est un tournoi sans diamant fortement connexe. Nous montrons comme dans $c_{2}$ que $R^{\prime} /(E-Y \cup J) \sim R /(E-Y \cup J)$, et par suite $R / C \sim R^{\prime} / C$.

$\left.c_{4}\right)$ Si $|Y| \leq 3$.

Soient $J$ une partie de $K$ tel que $|Y|+|J|=6$ et $R /(K-J)$ est un tournoi sans diamant fortement connexe. Nous montrons comme dans $c_{2}$ que $R^{\prime} /(E-Y \cup J) \sim R /(E-Y \cup J)$, et par suite $R / C \sim R^{\prime} / C$.

ii) Si $R / K$ est une chaîne, on a trivialement $R / K$ et $R^{\prime} / K$ sont $(\leq 6)$ hypomorphes. Dans la suite on suppose que $R / K$ est un tournoi sans diamant fortement connexe et $6 \leq|K| \leq 9$. Comme $K \neq D$, alors le i) du 
lemme 2.22 prouve que $R / K$ et $R^{\prime} / K$ sont $(\leq 5)$-hypomorphes. Suivant le cardinal de $K$, on a :

$\left.c_{1}\right) \mathrm{Si}|K|=6$. Comme $K$ est différente de sa composante, alors le lemme 2.12 prouve que $R^{\prime} / K \sim R / K$, d'autre part $\left(R / K\right.$ et $\left.R^{\prime} / K\right)$ sont ( $\leq 5)$-hypomorphes. Il s'ensuit que $R / K$ et $R^{\prime} / K$ sont $(\leq 6)$-hypomorphes.

$\left.c_{2}\right) \mathrm{Si}|K|=7$. Soient $A$ une partie de $K$ de cardinal 6 et $\{a\}=K-A$. Les relations $R /(E-\{a\})$ et $R^{\prime} /(E-\{a\})$ sont $(-5)$-hémimorphes et $A$ est différente de sa composante, alors le lemme 2.12 prouve que $R / A \sim R^{\prime} / A$. Il s'ensuit que $R / K$ et $R^{\prime} / K$ sont $(\leq 6)$-hypomorphes.

$\left.c_{3}\right) \mathrm{Si}|K|=8$. Soient $A$ une partie de $K$ de cardinal 6 et $\{a, b\}=K-A$. Les relations $R /(E-\{a, b\})$ et $R^{\prime} /(E-\{a, b\})$ sont (-4)-hémimorphes. Si $R / A$ est une chaine, on a $R / A \sim R^{\prime} / A$. Si $R / A$ est fortement connexe. Soient $H=R /(\{x\} \cup A)$, et $(y, z, t) \in A^{3}$ avec $R /\{y, z, t\}$ est un 3-cycle. Considérons alors les parties $F$ de $E$ contenant $\{x, y, z, t\}$ et telles que $: R / F \sim H$ ou $R / F \sim H^{\star}$. Il n'y en a pas d'autre que $\{x\} \cup A$, car $\{x\}$ et $A$ sont les composantes fortement connexes de $H=R /(\{x\} \cup A)$. Le nombre de parties $F$ de $E$ contenant $\{x, y, z, t\}$ telle que $R / F$ est hémimorphe à $H$ est donc égal à 1 (c.a.d $n(R /(E-\{a, b\}), H,\{x, y, z, t\})=$ 1 ). D'après le lemme 2.7 , le nombre de parties $F$ de $E$ contenant $\{x, y, z, t\}$ telles que $R^{\prime} / F$ est hémimorphe à $H$ est donc égal à 1 (c.a.d $n\left(R^{\prime} /(E-\right.$ $\{a, b\}), H,\{x, y, z, t\})=1)$. Il s'ensuit que $R^{\prime} / A \sim R / A$, et par suite $(R / K$ et $\left.R^{\prime} / K\right)$ sont $(\leq 6)$-hypomorphes.

$\left.c_{4}\right) \mathrm{Si}|K|=9$. Soient $A$ une partie de $K$ de cardinal 6 et $\{a, b, c\}=$ $K-A$. Les relations $R /(E-\{a, b, c\})$ et $R^{\prime} /(E-\{a, b, c\})$ sont $(-3)$ hémimorphes. Si $R / A$ est une chaine, on a $R / A \sim R^{\prime} / A$. Si $R / A$ est fortement connexe. Soient $H=R /(\{x\} \cup A)$, et $(y, z, t) \in A^{3}$ avec $R /\{y, z, t\}$ est un 3-cycle. Considérons alors les parties $F$ de $E$ contenant $\{x, y, z\}$ et telles que : $R / F \sim H$ ou $R / F \sim H^{\star}$. Il n'y en a pas d'autre que $\{x\} \cup A$, car $\{x\}$ et $A$ sont les composantes fortement connexes de $H=R /(\{x\} \cup A)$. Le nombre de parties $F$ de $E$ contenant $\{x, y, z\}$ telle que $R / F$ est hémimorphe à $H$ est donc égal à 1 (c.a.d $n(R /(E-\{a, b, c\}), H,\{x, y, z\})=1)$. D'après le lemme 2.7, le nombre de parties $F$ de $E$ contenant $\{x, y, z\}$ telles que $R^{\prime} / F$ est hémimorphe à $H$ est donc égal à 1 (c.a.d $n\left(R^{\prime} /(E-\{a, b, c\}), H,\{x, y, z\}\right)=$ $1)$. Il s'ensuit que $R^{\prime} / A \sim R / A$, et par suite $\left(R / K\right.$ et $\left.R^{\prime} / K\right)$ sont $(\leq 6)$ hypomorphes.

Lemme 8.8. Si $h \geq 4$ et $|D| \geq 6+h$ et si $\left(R / D\right.$ et $\left.R^{\prime} / D\right)$ sont $(\leq$ $6)$-hypomorphes, alors il existe une partie $A$ de $D$ de cardinal $h$ tel que $R /(D-A)$ et $R^{\prime} /(D-A)$ sont isomorphes mais non anti-isomorphes. 
Preuve. La preuve est analogue à la preuve du lemme 7.7.

Lemme 8.9. $R / C \sim R^{\prime} / C$.

Preuve. Posons $X=E-(C \cup D)$. D'après le lemme 8.5 on peut supposer que $|X| \leq 5$, de même d'après le lemme 8.6 on suppose que $|E-D| \leq 11$, et par suite $|C| \leq 11$, aussi d'après le lemme 8.7 on suppose que $\left(R / D\right.$ et $\left.R^{\prime} / D\right)$ sont $(\leq 6)$-hypomorphes. Suivant le cardinal de $X$, on a:

$\left.c_{1}\right)|X|=0$. D'après le lemme 8.8 il existe une partie $A$ de $D$ de cardinal 6 telle que $R /(D-A)$ et $R^{\prime} /(D-A)$ sont isomorphes mais non anti-isomorphes. Soit $f$ un hémimorphisme de $R /(E-A)$ sur $R^{\prime} /(E-A)$.

- Si $f$ est un isomorphisme, comme $R^{\prime} /(D-A) \sim R /(D-A)$, alors le lemme 2.6 prouve que $R / C \sim R^{\prime} / C$.

- Si $f$ est un anti-isomorphisme. D'après le lemme 8.4 $R^{\prime} / C \sim R / C$ ou $R^{\prime} / C \sim R^{\star} / C$. Si $R^{\prime} / C \sim R^{\star} / C$, alors d'après le lemme $2.6 R^{\prime} /(D-A) \sim$ $R^{\star} /(D-A)$, ce qui est impossible. Donc $R / C \sim R^{\prime} / C$.

$\left.c_{2}\right)|X|=1$. D'après le lemme 8.8 il existe une partie $A$ de $D$ de cardinal 5 telle que $R /(D-A)$ et $R^{\prime} /(D-A)$ sont isomorphes mais non antiisomorphes. Soit $f$ un hémimorphisme de $R /(E-A \cup X)$ sur $R^{\prime} /(E-A \cup X)$.

- Si $f$ est un isomorphisme, comme $R^{\prime} /(D-A) \sim R /(D-A)$, alors le lemme 2.6 prouve que $R / C \sim R^{\prime} / C$.

- Si $f$ est un anti-isomorphisme. D'après le lemme 8.4 $R^{\prime} / C \sim R / C$ ou $R^{\prime} / C \sim R^{\star} / C$. Si $R^{\prime} / C \sim R^{\star} / C$, alors d'après le lemme $2.6 R^{\prime} /(D-A) \sim$ $R^{\star} /(D-A)$, ce qui est impossible. Donc $R / C \sim R^{\prime} / C$.

$\left.c_{3}\right)|X|=2$. D'après le lemme 8.8 il existe une partie $A$ de $D$ de cardinal 4 telle que $R /(D-A)$ et $R^{\prime} /(D-A)$ sont isomorphes mais non antiisomorphes. Soit $f$ un hémimorphisme de $R /(E-A \cup X)$ sur $R^{\prime} /(E-A \cup X)$. Nous montrons comme dans $c_{2}$ que $R / C \sim R^{\prime} / C$.

$\left.c_{4}\right) 3 \leq|X| \leq 5$. D'après le lemme 8.8 il existe une partie $A$ de $D$ de cardinal 4 telle que $R /(D-A)$ et $R^{\prime} /(D-A)$ sont isomorphes mais non anti-isomorphes. Soient $B$ une partie de $X$ de cardinal 2 et $f$ un hémimorphisme de $R /(E-A \cup X \cup B)$ sur $R^{\prime} /(E-A \cup X \cup B)$. Nous montrons comme dans $c_{2}$ que $R / C \sim R^{\prime} / C$. 


\section{References}

[1] Y. BOUDABBOUS et J. DAMMAK : Sur la (-k)-demi- reconstructibilité des tournois finis, CRAS, Série I 326, pp. 1037-1040, (1998).

[2] A. BOUSSAIRI : Thèse de doctorat de mathématiques. Soutenue à l'Université Claude Bernard, le 12 Juin, (1995).

[3] J. DAMMAK : Caractérisation des relations binaires finies d-demireconstructibiles. Proyecciones, Volume 22, N 1, (2003).

[4] J. DAMMAK : La dualité dans la demi-reconstruction des relations binaires finies, CRAS, Série I 327, pp. 861-864, (1998).

[5] J. DAMMAK : La (-5)-demi-reconstructibilité des relations binaires connexes finies. Proyecciones, Volume 22, N 3, (2003).

[6] R. FRAÏSSÉ : L'intervalle en théorie des relations, in orders, descriptions and roles, M. Pouzet et D. Richard éd. North-Holland, pp. 313342 , (1984).

[7] C. GNANVO et P.ILLE : La reconstruction des tournois. C. R. Acad. Sci. Paris, t. 306, série I, pp. 577-580, (1988).

[8] J. G. HAGENDORF et G. LOPEZ : La demi-reconstructibilité des relations binaires d'au moins 13 éléments. C. R. Acad. Sci. Paris, série I, 317, pp. 7-12, (1993).

[9] G. LOPEZ : L'indéformabilité des relations et multirelations binaires. Zeitschrift. Math. Logik Grundlagen Math. 24, pp. 303-317, (1978).

[10] G. LOPEZ et C. RAUZY : Reconstruction of binary relations from their restrictions of cardinality 2,3,4 and (n-1), I. Zeitschr. f. Math. Logik und Grundlagen d. Math. Bd. 38, S. 27-37, (1992).

[11] G. LOPEZ et C. RAUZY : Reconstruction of binary relations from their restrictions of cardinality 2,3,4 and (n-1), II. Zeitschr. f. Math. Logik und Grundlagen d. Math. Bd 38, S. 157-168, (1992). 
[12] M. POUZET : Application d'une propriété combinatoire des parties d'un ensemble aux groupes et aux relations. Math. Zeitschr. 150, pp. 117-134, (1976).

Received : April, 2003.

\section{Jamel Dammak}

Département de Mathématiques

Faculté des Sciences de Sfax

Université Claude Bernard Lyon 1

B. P. 802, 3018 Sfax

Tunisie

e-mail : jdammak@yahoo.fr 